Conclusion Perceived work-related mental health is greatly affected by age and gender. Improving mentally healthy working environment could be more effective if workers' age and gender are taken into account. Future studies are needed to identify any difference of demographic characteristics between self-reported work-mental health issues by workers and diagnosed mental illness resulting from work by doctors for better workplace health and safety interventions.

\section{RF-270 DOES THE TIMING AND DURATION OF MENTAL HEALTH PROBLEMS DURING CHILDHOOD AND ADOLESCENCE MATTER FOR LABOUR MARKET PARTICIPATION OF YOUNG ADULTS?}

${ }^{1}$ Samira de Groot. 'University of Groningen - University Medical Centre Groningen, Netherlands

\subsection{6/OEM-2021-EPI.379}

Introduction Little is known about the effect of timing and duration of mental health problems (MHPs) on young adults' labour market participation (LMP).

Objective This life-course study aims to examine whether and how the timing and duration of MHPs between ages 11 to 22 are associated with LMP at age 26.

Methods Data was used from Tracking Adolescents' Individual Lives Survey (TRAILS), an ongoing Dutch prospective cohort study. Internalising and externalising problems were measured by the Youth/Adult Self-Report at ages 11, 13, 16, 19 and 22. LMP (having a paid job yes/no) was assessed at age 26. Logistic regression analyses were performed with 15 -year follow-up data $(\mathrm{N}=874)$.

Results Internalising problems at all ages and externalising problems at ages 13,19 and 22 were associated with an increased risk of not having a paid job (internalising problems odds ratios [ORs] ranged from 2.24, 95\% confidence interval [CI] $1.02-4.90$ at age 11 to OR 6.58 , CI $3.14-13.80$ at age 22 ; externalising problems ORs from 2.84, CI 1.11-7.27 at age 13 to OR 6.36, CI 2.30-17.56 at age 22). Especially young adults with a long duration of internalising problems had an increased risk of not having a paid job in young adulthood compared with participants without mental health problems (OR 3.20, CI 1.88-5.45).

Conclusion Regardless of the timing, experiencing MHPs during childhood or adolescence increases the risk of not having a paid job. In particular, those young adults with a long duration of internalising problems are at risk of not having a paid job. These findings emphasize the necessity of applying a lifecourse perspective when investigating the effect of MHPs on LMP. Early monitoring, mental health care, and timely provision of employment support may improve young adult's participation in the labour market.

\section{RF-382 ASSOCIATION BETWEEN WORKPLACE BULLYING AND ACUTE AND CHRONIC LOW BACK PAIN IN CIVIL SERVANTS FROM A MIDDLE-INCOME COUNTRY}

${ }^{1}$ Fernando Feijó, Neil Pearce, Neice Faria, Maitê Carvalho, Ana Laura Szortyka, Paulo Oliveira, Anaclaudia Fassa. 'Federal University of Bahia, Brazil
Introduction Psychosocial factors at work are risk factors for several health outcomes, including musculoskeletal disorders such as low back pain (LBP). Few studies from high-income countries suggest a positive association between bullying at work and musculoskeletal pain, but the knowledge on this relationship is scarce. None studies on the theme are available from low- and middle-income countries.

Objectives Therefore, we aimed to investigate the association between workplace bullying and LBP in the last 7 days and chronic LBP in civil servants from a middle-income country.

Methods This is a cross-sectional study with 894 participants from judicial civil servants from a city in southern Brazil. Workplace Bullying was measured by the Negative Acts Questionnaire (NAQ-r) and Low Back Pain by the Nordic Questionnaire for Musculoskeletal Symptoms (NQMS). Logistic Regression was used to analyse data and test hypotheses, estimating prevalence odds ratios (POR).

Results The prevalence of workplace bullying was $18.2 \%$. The overall prevalence of LBP in the last 7 days was $50.1 \%$, and of Chronic LBP was $19.3 \%$. Psychosocial factors at work such as occupational stress were strongly associated with both outcomes. Workplace bullying was strongly associated with LBP, even after adjustment for several confounders. The risk of LBP in the last 7 days among bullied workers was $89.0 \%$ higher $(\mathrm{POR}=1.89$; 95\% $\mathrm{CI}$ : 1.31-2.71), compared to non-bullied employees. Bullying was also associated with chronic LBP after adjustment for sociodemographic, behavioural and some occupational factors $(\mathrm{POR}=1.60 ; 95 \% \mathrm{CI}$ : 1.05-2.44).

Conclusions Psychosocial factors at work, particularly workplace bullying, were strong risk factors for LBP, in contrast to most individual factors. Dose-response patterns were showed. Positive associations between bullying and LBP raise hypotheses on causation, emphasising the role of bullying as an important psychosocial risk at work. Further longitudinal studies should address these hypotheses, investigating mechanisms, effect modification and possible mediation.

\section{Respiratory}

\section{RF-112 ASBESTOS-RELATED DISEASE IN THE ONTARIO ASBESTOS WORKERS REGISTER}

${ }^{1}$ Victoria H Arrandale, Paul Demers, Colin Berriault, Leon Genesove, Nathan DeBono, Chaojie Song. 'University of Toronto, Canada

\subsection{6/OEM-2021-EPI.381}

Objectives Research on asbestos-related disease (ARD) has traditionally focused on groups with well-recognized, consistently high exposure. Contemporary exposure to asbestos occurs more intermittently, for example during maintenance of asbestos-containing materials (ACM). The Ontario Asbestos Workers Register (AWR) was established in 1986 to track asbestos exposure. This study reports on the risk of ARD among workers in the AWR.

Methods Overall 33,490 AWR registrants were included. Data were linked probabilistically with administrative health databases (1986-2018) to identify cases of ARD. Workers were followed until diagnosis, death, age 100, last contact with the health system, or end of study, whichever occurred first. Incidence rates were compared to the general population using standardized incidence ratios (SIRs). Associations between 
ACM exposure and ARD were estimated using Poisson regression.

Results Overall 26,401(79\%) AWR registrants were linked to health data; $97 \%$ were male. The mean age at cohort entry was 36 years. The most common industries represented were construction (61\%), manufacturing (20\%) and education (10\%). Among men and women rates were markedly increased for mesothelioma [M:SIR 6.55(95\% CI=5.34-7.96); W:SIR 19.3 (3.87-56.3)] and pulmonary fibrosis [M:SIR 13.8(11.9-15.9); W:SIR 9.15(2.46-23.4)]. The risk of asbestosis was also elevated, but did not reach statistical significance among women [M:SIR 11.1(9.46-12.9); W:SIR 1.21(0.02-6.75)]. Among men and women, rates of COPD and lung cancer were elevated, but few cases were observed among women. Compared to workers with 238 hours had increased rates of lung cancer [RR 1.3(1.07-1.57)], mesothelioma [RR 3.13(1.945.06)], asbestosis [RR 3.31(2.33-4.71)], COPD [RR 1.34 (1.22-1.48)], and pulmonary fibrosis [RR 1.86(1.34-2.58)].

Conclusion Exposure to asbestos in construction and building maintenance continues to contribute to ARD incidence. Though a Canadian ban on asbestos use in new products is encouraging, it is likely to have minimal impact on ARD resulting from construction activities, where exposure to existing ACM will persist.

\section{RF-274 CARDIORESPIRATORY FITNESS OF POLICE RECRUITS: NORM-REFERENCED VALUES AND 14-YEAR TEMPORAL TREND.}

'Sébastien Poirier, Julie Houle, François Trudeau. 'Université du Québec à Trois-Rivières, Canada

\subsection{6/OEM-2021-EPI.382}

Introduction Many have highlighted the increased risk of cardiovascular diseases (CVD) among police officers. Because a low cardiorespiratory fitness (CRF) in young adulthood is associated with a higher risk of CVD incidence, the identification of police officers exhibiting low CRF upon entry in the profession could allow the implementation of targeted strategies for the primary prevention of CVD. Unfortunately, reference values for the CRF of Canadian police recruits are not yet available.

Objectives This research aimed to provide reference standards for the CRF of police recruits in Québec (Canada) and to evaluate the trend in the CRF of recruits over a 14-year period.

Methods This cross-sectional study was divided into two research phases. First, fifty-one police cadets performed both an indirect calorimetry assessment of their maximal oxygen consumption (VO2max) and the Physical Abilities Test (PAT) used for the recruitment of police officers in the province of Québec. Using results from both tests, a model was developed to estimate $\mathrm{VO}_{2}$ max based on PAT results. The CRF of police recruits who completed the PAT from 2004 to 2017 was then retrospectively assessed using the previously developed model. Results Based on the analysis of 7234 PAT results, the average $\dot{\mathrm{V}} \mathrm{O}_{2} \max$ was $53.33 \pm 2.90 \mathrm{ml} \cdot \mathrm{kg}^{-1} \cdot \mathrm{min}^{-1}$ for male recruits and $43.78 \pm 2.89 \mathrm{ml} \cdot \mathrm{kg}^{-1} \cdot \mathrm{min}^{-1}$ for female recruits. Furthermore, bivariate correlation analyses suggested small but significant increases in the $\dot{\mathrm{VO}_{2}}$ max of both male $\left(\mathrm{r}_{\mathrm{s}}=.105, \mathrm{p}<\right.$ $.001)$ and female recruits $\left(r_{s}=.125, p<.001\right)$ over the 14 year period studied.
Conclusion Our results suggest that the CRF of police recruits in Québec is considerably higher than their North American peers. Furthermore, although clear conclusions cannot be drawn from our temporal trend analyses, our data suggest that the CRF of police officers from this population did not decline over the last decades as previously suggested by others.

\section{RF-408 WORK-RELATED RESPIRATORY SYMPTOMS AND ECZEMA AMONG SWEDISH EYELASH STYLISTS}

${ }^{1}$ Sara Gunnare, Helen Wahlkvist. ' Karolinska Institutet, Sweden

\subsection{6/OEM-2021-EPI.383}

Introduction Eyelash extension is a beauty treatment established in recent years. The eyelash stylists apply eyelash extensions on natural lashes, using adhesives based on cyanoacrylates. Exposure to cyanoacrylates may cause asthma and eczema.

Objectives The main objective was to survey work-related respiratory symptoms and eczema among eyelash stylists in Sweden. Occupational exposure and use of personal protective equipment were also studied.

Methods Questionnaires with focus on work-related respiratory symptoms, eczema and occupational exposure conditions were sent to 656 eyelash stylists in the four largest cities of Sweden (Stockholm, Göteborg, Malmö and Uppsala). The participants were identified by an internet reservation site for eyelash extensions and by social media. The participation rate was 26\% (169 respondents).

Results The main part of the respondents (74\%) had been working as eyelash stylists for more than 2 years. 59\% of them reported that they had respiratory symptoms and 15\% that they had eczema (12-month prevalence). One third of the respondents had a colleague who had changed occupations due to respiratory symptoms or eczema. Almost 20\% reported that they had customers who had had respiratory symptoms in association with lash extension and almost $10 \%$ stated that customers got eczema. Approximately $85 \%$ of the respondents reported that they used personal protective equipment, but more than $50 \%$ only used surgical masks that do not protect against acrylates. About 20\% reported that they were using protective gloves at work.

Conclusion The survey shows that many eyelash stylists have respiratory symptoms and that some of them have eczema. The surgical masks most commonly used do not provide sufficient protection against acrylates present in the work environment of the eyelash stylists. In addition, the survey indicates that far too few eyelash stylists use protective gloves at work.

\section{RF-417 OCCUPATIONAL RHINITIS AND ASTHMA CAUSED BY ISOCYANATES}

${ }^{1}$ Siwar Chemingui, Jihen Hsinet, Saloua Ismail, Amani Dallagi, Nihel Khouja, Ines Aissa, Aida Benzarti, Abdelmajid Ben Jemaa. 'Hôpital La Rabta, Tunisia

\subsection{6/OEM-2021-EPI.384}

Introduction Isocyanates are strong respiratory sensitizers. Repeated and prolonged occupational exposure to these 